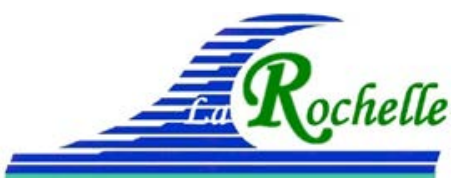

XV $V^{\text {èmes }}$ Journées Nationales Génie Côtier - Génie Civil La Rochelle, 29 au 31 mai 2018

DOI:10.5150/jngcgc.2018.064 ～(C) Editions Paralia CFL

disponible en ligne - http://www.paralia.fr - available online

\title{
Projet EMACOP : analyse économique de la production d'énergie renouvelable de systèmes houlomoteurs sur le site d'Esquibien (Finistère)
}

\author{
Bertrand MICHARD ${ }^{1}$, Sébastien BOULAND ${ }^{2}$, Philippe SERGENT ${ }^{3}$, \\ Sixtine NEUVEGLISE ${ }^{3,4,5}$, Virginie BAUDRY ${ }^{6}$
}

1. Cerema EMF, Technopôle Brest Iroise, BP 5, 29280 Plouzané, France. bertrand.michard@cerema.fr

2. École Nationale des Travaux Publics de l’État, 3 rue Maurice Audin, 69518 Vaulxen-Velin, France. sebastien.bouland@entpe.fr

3. Cerema EMF, 134 rue de Beauvais, CS 60039, 60280 Margny lès Compiègne Cedex, France.phillipe.sergent@cerema.fr ;sixtine.neuveglise@cerema.fr

4. Université de Technologie de Compiègne, 15 rue Roger Couttolenc, 60200 Compiègne, France.

5. Université Le Havre Normandie, 25 rue Philippe Lebon, 76600 Le Havre, France

6. École Centrale Nantes, 1 Rue de la Noë, 44300 Nantes, France.

virginie.baudry@ec-nantes.fr

\section{Résumé :}

Un des objectifs du projet de recherche EMACOP (Energies MArines, COtières et Portuaires) est de caractériser le potentiel houlomoteur près des structures portuaires et côtières. L'article présente l'analyse économique de la production d'énergie de deux systèmes houlomoteurs oscillants sur le site d'Esquibien (Finistère). L'approche du calcul de coût de l'énergie et les principes des systèmes sont décrits pour le flotteur pilonnant bord à quai, modélisé par l'Université de Technologie de Compiègne, l'Université Le Havre Normandie et le Cerema (NEUVEGLISE et al., 2017), et pour le batteur inversé à proximité de la digue d'Esquibien, développé par l'École Centrale de Nantes (BAUDRY et al., 2015). La méthodologie de calcul de la production d'énergie repose sur la multiplication des matrices de puissance des dispositifs par des matrices de ressource, issues des simulations numériques du modèle SWASH (Simulating WAves till SHore) de transformation de vagues en proche côtier (MICHARD et al., 2016). Les résultats obtenus, associés aux coûts d'investissement et de fonctionnement des systèmes, permettent d'évaluer les coûts de l'énergie sur des durées d'exploitation de 20 et 30 ans. Ces coûts, qui restent néanmoins difficiles à évaluer précisément en raison de technologies innovantes et d'incertitudes liées à la variabilité de la ressource, sont du même ordre de grandeur que ceux des systèmes éoliens offshore référencés .

Mots-clés : Énergie, Marine, Renouvelable, EMR, Système houlomoteur, oscillant, flotteur pilonnant, batteur inversé, Esquibien, Finistère, EMACOP. 


\section{Thème 5 - Énergies et ressources marines}

\section{Introduction}

La Loi de transition énergétique de 2015, qui prévoit de diviser par quatre les émissions de gaz à effet de serre en 2050 par rapport aux niveaux de l'année 1990, implique un développement important des énergies marines renouvelables en France dans les années à venir. Le Projet National de Recherche EMACOP (Énergies MArines, CÔtières et Portuaires) a pour objectifs d'étudier et de promouvoir le développement de systèmes de récupération d'énergie marine adaptés aux structures côtières et portuaires le long des côtes françaises. Un des objectifs du thème S2 intitulé "Systèmes houlomoteurs sur ouvrages anciens » est de caractériser le potentiel houlomoteur près de ces structures. En tranche 3 de projet, le travail a porté sur l'analyse économique de la production d'énergie renouvelable de systèmes houlomoteurs oscillants sur le site d'Esquibien retenu en tranche 1 (MICHARD et al., 2014). Il s'agit d'évaluer les coûts de l'énergie pour des systèmes bord à quai ou positionné à proximité de la digue. L’approche du calcul de coût de l'énergie et les principes des systèmes sont présentés pour le flotteur pilonnant bord à quai, modélisé par l’Université de Technologie de Compiègne, l'Université Le Havre Normandie et le Cerema (NEUVEGLISE et al., 2017), et pour le batteur inversé à proximité de la digue, développé par l’École Centrale de Nantes (BAUDRY et al., 2015). Le système à flotteur pilonnant a été optimisé au niveau du PTO (Power Take-Off) et de la géométrie du flotteur, afin d'obtenir des matrices de puissance adaptées au cas réel de la digue d’Esquibien, tandis que l'énergie récupérée par le système à batteur inversé a été estimée sous la forme d’une matrice de puissance. La méthodologie de calcul de la production d'énergie repose sur la multiplication des matrices de puissance des dispositifs par des matrices de ressource, issues des simulations numériques du modèle de transformation de vagues en proche côtier SWASH (Simulating WAves till SHore) du point de sortie $n^{\circ} 1$ pour le système bord à quai et du point $\mathrm{n}^{\circ} 4$ pour le système positionné à proximité de la digue (voir figures 1 et 2) de la tranche 2 (MICHARD et al., 2016).

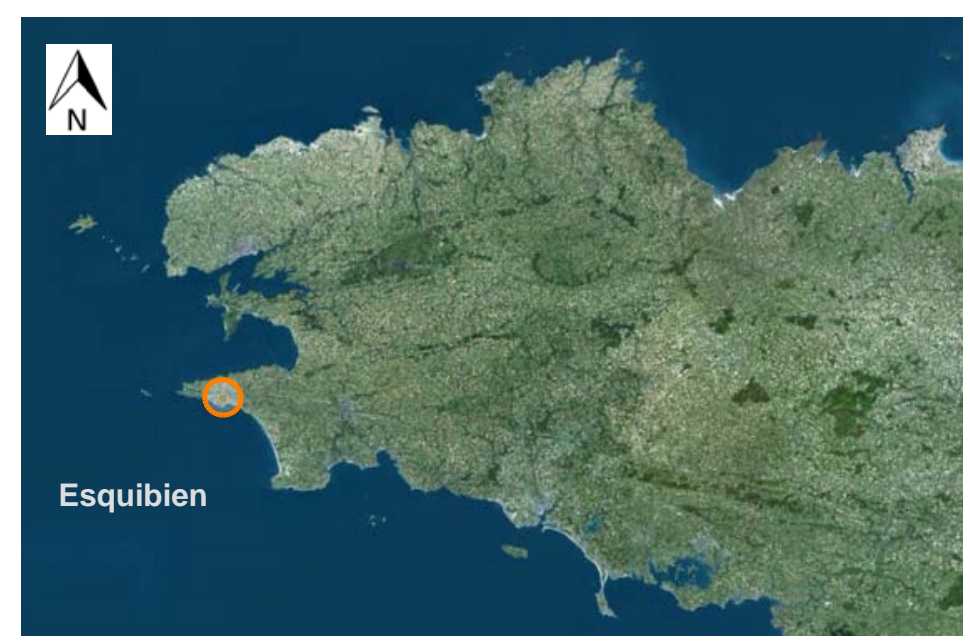

Figure 1. Localisation d'Esquibien, Finistère, France (http://cms.geobretagne.fr/). 


\section{XVèmes Journées Nationales Génie Côtier - Génie Civil La Rochelle, 29 au 31 mai 2018}

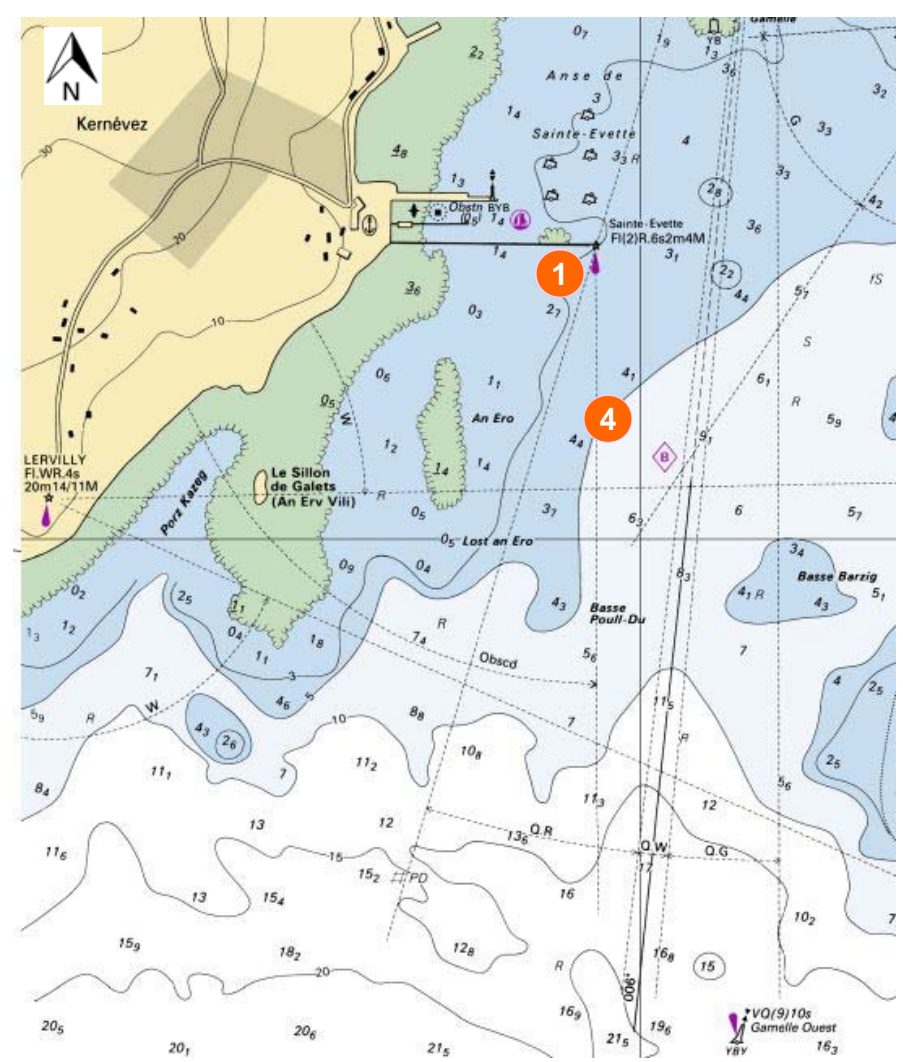

Figure 2. Emplacements des points de sortie $n^{\circ} 1$ (3 $\mathrm{m} \mathrm{CM}$ ) et $n^{\circ} 4(5 \mathrm{~m} \mathrm{CM})$ issus des simulations numériques SWASH réalisées en tranche 2 de projet sur le site d'Esquibien, constituant les matrices de ressource (carte marine $n^{\circ}$ 7147, www.datashom.fr)

\section{Définitions et principes}

\subsection{Indicateur du coût de l'énergie et approche du calcul de coût}

Le coût de l'énergie peut être défini par l'indicateur COE (Cost Of Energy) en $€ / \mathrm{kWh}$, qui prend en compte le coût initial, le taux d'actualisation et les coûts d'exploitation par rapport à l'énergie produite sur la durée d'exploitation (voir figure 3). Le coût initial assimilé au CAPEX (CAPital EXpenditure) comprend les coûts d'études, de projet, de construction, de procédures, de pilotage et de coordination. Évaluer ce coût est un exercice délicat, car il dépend du type d'installation, de l'emplacement et des caractéristiques particulières du projet. Ce coût comprend le prix d'achat du système houlomoteur, son installation, ses coûts d'amarrage et d'installation électrique et de raccordements marin et terrestre. À ce coût, il faut ajouter le coût de démantèlement et les coûts de réparation et maintenance, assimilés à l'OPEX (OPerational EXpenditure), pour obtenir le coût total du système (équation 1 ) :

$$
\mathrm{C}_{\text {total Système }}=\underbrace{\mathrm{C}_{\text {initial }}}_{\text {CAPEX }}+\mathrm{C}_{\text {démantèlement }}+\underbrace{\left(\mathrm{C}_{\text {reparation }}+\mathrm{C}_{\text {maintenance }}\right)}_{\text {OPEX }}
$$




\section{Thème 5 -Énergies et ressources marines}

Une des difficultés concerne l'estimation des coûts de réparation et de maintenance, liés à la fréquence des événements extrêmes et à la fatigue des systèmes; en effet, les systèmes bord à quai sont encore au stade expérimental et les retours d'expérience restent limités. Pour ces technologies innovantes, et considérées comme peu matures, un taux d'actualisation de $10 \%$ sur une durée de 20 ans et de $8 \%$ sur 30 ans sont retenus dans l'analyse en cohérence avec les pratiques actuelles (EQUIMAR, 2011; GUANCHE et al., 2014 ; ADEME, 2016).

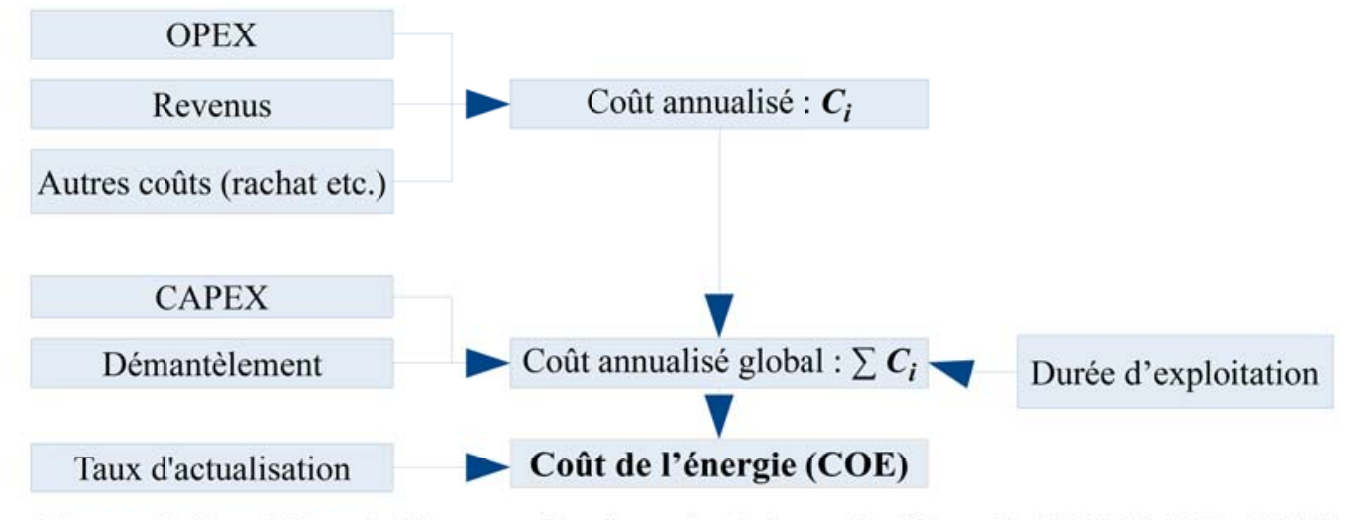

Figure 3. Synthèse de l'approche du calcul de coût (d'après EQUIMAR, 2011).

Le coût de l'énergie dépend en particulier des caractéristiques du site, c'est-à-dire la ressource énergétique des vagues et l'accessibilité des systèmes. Les systèmes ont des exigences distinctes qui conditionnent le coût de l'énergie produite. Ainsi, le coût initial du système à flotteur pilonnant dépend de l'état de l'ouvrage et de la proximité du réseau électrique, tandis que pour le système à batteur inversé, la profondeur d'eau optimale peut se situer à plusieurs centaines de mètres au large, rendant les opérations de réparation et maintenance onéreuses.

\subsection{Principes des systèmes houlomoteurs}

Il s'agit d'estimer le rendement et le coût de l'énergie produite par les deux systèmes houlomoteurs, ayant pour principe de transformer l'énergie mécanique, composée des énergies cinétique et potentielle, en énergie électrique. Ainsi, la capacité d'absorption de l'énergie des vagues et la notion de rendement des systèmes houlomoteurs sont des paramètres importants à considérer dans l'analyse économique.

a) Système oscillant à flotteur pilonnant

Les caractéristiques géométriques du dispositif développé par l'UTC-ULHN-Cerema (NEUVEGLISE et al., 2017) sont les dimensions de la demi-largeur du flotteur $l$, du tirant d'eau $\Gamma$ et de la distance de dégagement $D$ par rapport à la digue (voir figure 4). La conversion d'énergie dépend aussi des paramètres de houle (hauteur significative $H s$ et période de pic $T p$ ) et de la profondeur d'eau $d$ au point d'installation du dispositif. L'optimisation géométrique du flotteur passe par la variation de la largeur, et plus précisément de sa demi-largeur, et par son tirant d'eau, qui n'a pas été traite ici. Ainsi, 


\section{XVèmes Journées Nationales Génie Côtier - Génie Civil La Rochelle, 29 au 31 mai 2018}

pour évaluer la pertinence de l'optimisation de la largeur, il est nécessaire de calculer l'énergie annuelle produite pour différentes largeurs de flotteur. L'hypothèse d'un flotteur de forme rectangulaire aux dimensions métriques, constitué en matériaux composites relativement légers, a conduit à supposer que le tirant d'eau de ce flotteur est inférieur au mètre ; le choix d'un tirant d'eau constant de $0,75 \mathrm{~m}$ a donc été fait pour les calculs de puissance de houle.

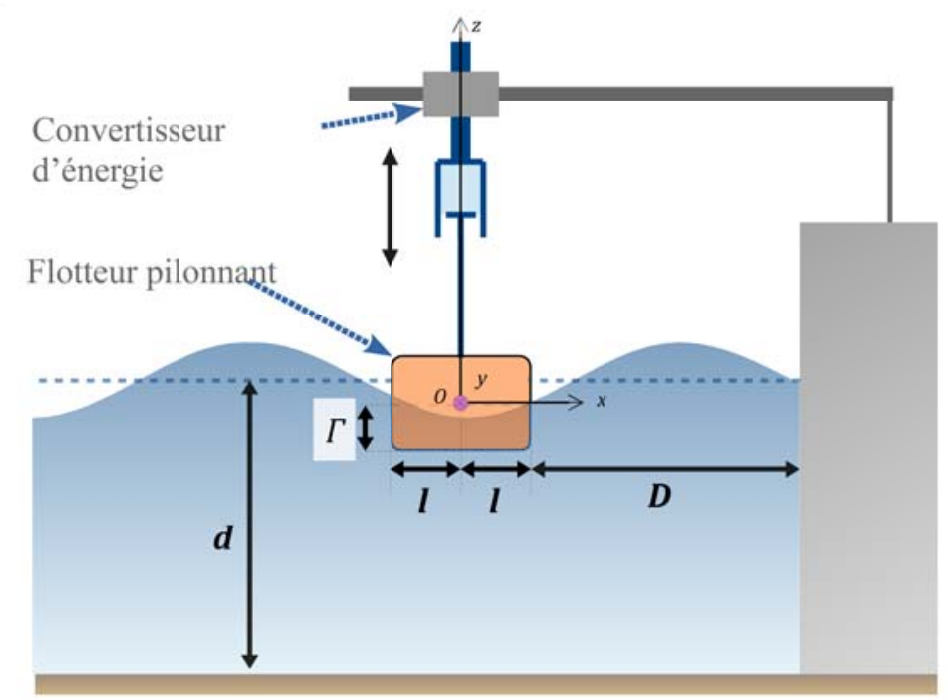

Figure 4. Principe et caractéristiques du système oscillant de type flotteur pilonnant.

b) Système oscillant à batteur inversé

L'évaluation du rendement du système partiellement immergé et perçant la surface libre (voir figure 5) a été réalisé par l'ECN (BAUDRY et al., 2015). Les résultats transmis prennent la forme d'une matrice de puissance rassemblant, pour les couples de houle $(H s, T p)$ donnés, les puissances produites en $\mathrm{kW}$. Les dimensions du système sont les suivantes : largeur $l$ de $20 \mathrm{~m}$, hauteur $h$ de $13 \mathrm{~m}$, avec la hauteur de flap de $11 \mathrm{~m}$, épaisseur de flap de 1,5 m, masse d'acier de 60 tonnes et épaisseur d'acier de $15 \mathrm{~mm}$.

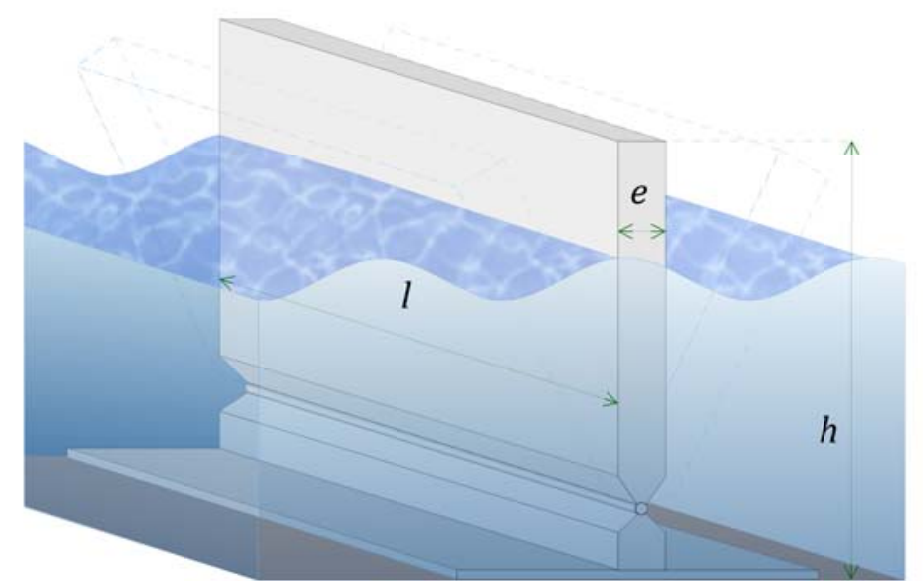

Figure 5. Description du système oscillant de type batteur inversé (flap oscillant seul) 


\section{Thème 5 -Énergies et ressources marines}

\section{Méthodologie}

Pour estimer les coûts de production de l'énergie sur le site, il faut croiser les matrices d'occurrence des climats de houle obtenues en tranche 2 de projet aux points de sortie $\mathrm{n}^{\circ} 1$ et $\mathrm{n}^{\circ} 4$, et les matrices de puissance produites par les systèmes à flotteur pilonnant et à batteur inversé (voir figure 6). L'énergie annuelle est calculée pour les conditions de houle dominante et trois niveaux de marée. L'estimation du coût de l'énergie est ensuite possible, en intégrant les coûts d'investissement CAPEX et de fonctionnement OPEX.

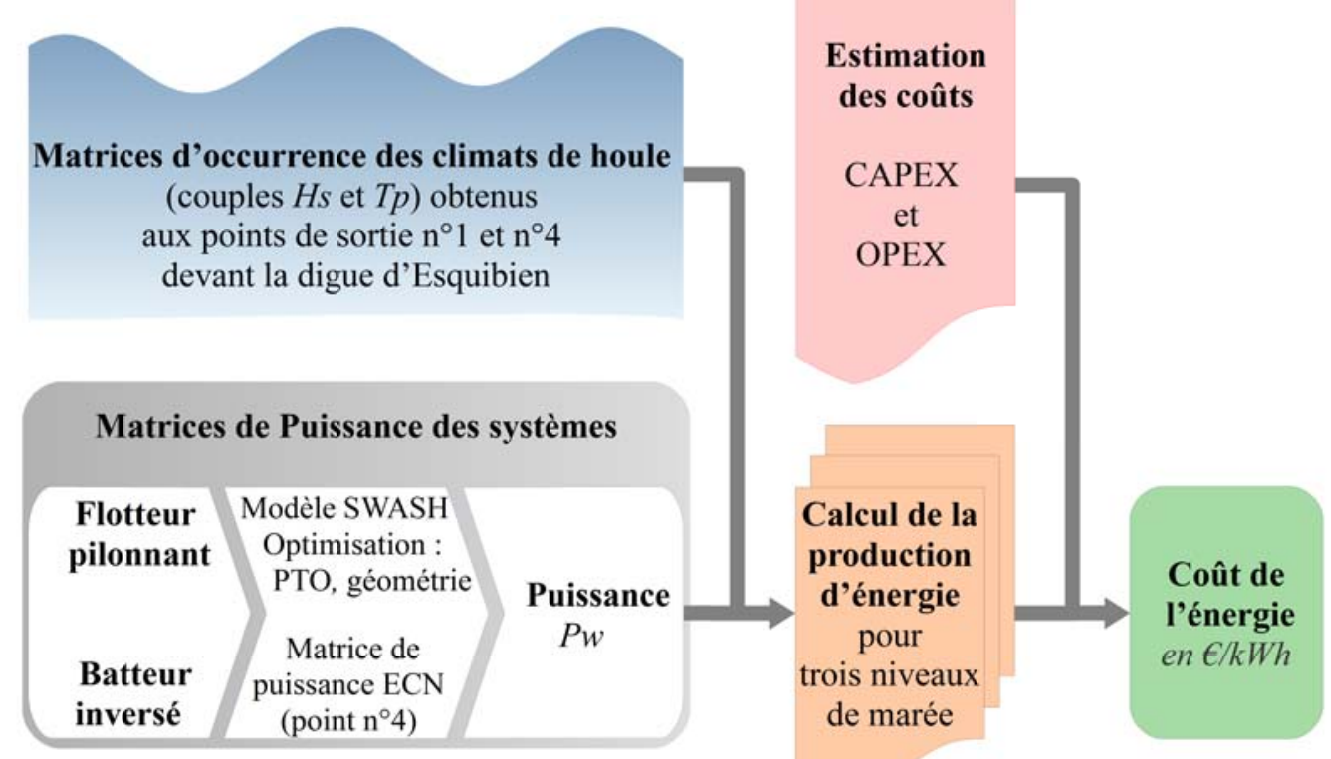

Figure 6. Méthodologie d'estimation des coûts de l'énergie des systèmes houlomoteurs

\subsection{Puissance des systèmes houlomoteurs}

a) Système oscillant à flotteur pilonnant

En exploitant l'application de l'UTC-ULHN-Cerema, et en optimisant les paramètres d'entrée, le coefficient d'extraction PTO (Power Take-Off) et la largeur du flotteur, il est possible de calculer pour chaque couple de houle $(H s, T p)$ associée à un niveau de marée, la puissance produite par le système modélisé. À cette étape, trois tableaux sont obtenus en présentant les puissances produites par les couples $(H s, T p)$ pour les niveaux d'eau de basse mer (1,45 m), mi-marée $(3,07 \mathrm{~m})$ et de pleine mer $(4,75 \mathrm{~m})$.

b) Système oscillant à batteur inversé

À partir de la matrice de puissance du batteur inversé de l'ECN, constitué d'un flap oscillant seul, de $20 \mathrm{~m}$ de long et positionné au point de sortie $\mathrm{n}^{\circ} 4$, il est possible de calculer la quantité d'énergie que ce système peut produire sur le site d'Esquibien.

\subsection{Calcul de la production d'énergie des systèmes}

a) Système oscillant à flotteur pilonnant

À partir des matrices d'occurrence des climats de houle et des matrices de puissance des 


\section{XVèmes Journées Nationales Génie Côtier - Génie Civil La Rochelle, 29 au 31 mai 2018}

systèmes, il est possible de calculer les matrices d'énergie en multipliant chaque couple de houle $(H s, T p)$ par son occurrence au point de sortie ${ }^{\circ} 1$. Il suffit ensuite de faire la somme de ces termes pour avoir l'énergie totale produite (RUSU et al., 2015). Le calcul des énergies est fait pour chaque niveau de marée, comme pour le calcul des puissances. Pour le cumul des trois niveaux de marée, une sommation est faite avec des coefficients de pondération ( 0,3 pour basse mer, 0,4 pour mi-marée et 0,3 pour pleine mer) afin de rassembler les trois tableaux d'énergie associés en un tableau global d'énergie annuelle.

b) Système oscillant à batteur inversé

Une approche similaire est proposée pour le système à batteur inversé. La matrice de puissance, correspondant au flap oscillant de $20 \mathrm{~m}$ de long, a été adaptée en utilisant les couples $(H s, T p)$, obtenus pour les trois niveaux de marée au point de sortie nº 4 .

\subsection{Coût de l'énergie}

Des estimations sont établies en tenant compte des coûts des systèmes à partir de la puissance du système, et des coûts d'autres dispositifs de types Wavedragon, Pelamis. Le coût du système d'amarrage, de fixation et de raccordement électrique sont pris en compte (CAPEX). Les coûts des opérations de maintenance (OPEX) doivent être inclus, même si ce calcul reste délicat en raison de retours d'expérience limités. À partir de ces éléments, en intégrant l'analyse des coûts du système Pelamis (DALTON et al., 2010), une estimation du coût initial des systèmes conduit à retenir $1 \mathrm{M€}$ pour le système à flotteur pilonnant (5 $\mathrm{m}$ de long, 2,5 $\mathrm{m}$ de demi-largeur), et de $5 \mathrm{M} €$ pour le système à batteur inversé (flap de $20 \mathrm{~m}$ de long, $11 \mathrm{~m}$ de haut et 1,5 m d'épaisseur).

\section{Résultats du système oscillant à flotteur pilonnnat}

\subsection{Quantité d'énergie produite}

Les résultats présentés ici concernent le cas d'un flotteur d'une demi-largeur de 2,5 m et d'un coefficient PTO adaptatif, implanté au niveau du point de sortie $\mathrm{n}^{\circ} 1$, positionné à une profondeur de $3 \mathrm{~m}$ CM près de la digue d'Esquibien. Les cases vertes des tableaux suivants 1 , 2 et 3 correspondent aux résultats de puissance produite par le système, et les valeurs voisines sont extrapolées. L'énergie annuelle est ensuite calculée en multipliant ces valeurs par les occurrences en nombre d'heures par an du climat de houle, ce qui fournit trois tableaux intermédiaires d'énergie annuelle non présentés ici. Pour le cumul des résultats, la sommation est réalisée avec les coefficients de pondération des niveaux de marée afin de rassembler les trois tableaux d'énergie correspondants en un tableau global d'énergie annuelle (voir tableau 4). Les résultats montrent que le système à flotteur pilonnant produit le plus d'énergie (8,34 MWh/an) pour le couple de houle ( $2 \mathrm{~m}, 12 \mathrm{~s}$ ). En sommant toutes les énergies produites pour les couples ( $H s, T p$ ), on obtient une énergie annuelle totale de 53,76 MWh par unité de longueur de flotteur, c'est-à-dire 1 m de long (voir tableau 5). 


\section{Thème 5 - Énergies et ressources marines}

Pour obtenir des résultats plus tangibles, une longueur de $5 \mathrm{~m}$ est choisie, multipliant la quantité d'énergie par 5, ce qui permet de produire une énergie totale de 268,8 Mwh/an.

Tableaux 1, 2, 3 et 4. Puissances produites (en $k W$ ) et énergies annuelles (en $M W h$ )

\begin{tabular}{|c|c|c|c|c|c|c|}
\hline \multicolumn{7}{|c|}{ 1. Puissances produites (kW) à basse mer } \\
\hline $\operatorname{Tp}(\mathrm{s})$ & 5 & 7 & 9 & 11 & 13 & 15 \\
\hline Hs (m) & 7 & 9 & 11 & 13 & 15 & 17 \\
\hline $4,5 \quad 5,5$ & & & & 10,47 & 12,94 & 15,41 \\
\hline $3,5 \quad 4,5$ & & & 7,59 & 9,17 & 10,48 & 11,79 \\
\hline $2,5 \quad 3,5$ & & 4,76 & 6,32 & 7,87 & 8,02 & 8,17 \\
\hline $1,5 \quad 2,5$ & 2,43 & 3,74 & 5,04 & 6,57 & 5,56 & 4,71 \\
\hline $0,5 \quad 1,5$ & 1,12 & 1,59 & 2,06 & 4,07 & 6,08 & \\
\hline
\end{tabular}

3. Puissances produites $(\mathrm{kW})$ à pleine mer

\begin{tabular}{|c|c|c|c|c|c|c|}
\hline $\operatorname{Tp}(\mathrm{s})$ & 5 & 7 & 9 & 11 & 13 & 15 \\
\hline Hs (m) & 7 & 9 & 11 & 13 & 15 & 17 \\
\hline $4,5 \quad 5,5$ & & & & 38,19 & 25,07 & 16,34 \\
\hline $3,5 \quad 4,5$ & & & 30,07 & 29,47 & 18,99 & 12,19 \\
\hline $2,5 \quad 3,5$ & & 20,21 & 20,48 & 20,75 & 12,91 & 8,03 \\
\hline $1,5 \quad 2,5$ & 0,21 & 5,55 & 10,89 & 12,03 & 6,83 & 3,88 \\
\hline $0,5 \quad 1,5$ & 0,00 & 1,56 & 3,17 & 3,53 & 3,89 & \\
\hline
\end{tabular}

\section{Puissances produites $(\mathrm{kW})$ à mi-marée}

\begin{tabular}{|c|c|c|c|c|c|c|}
\hline $\operatorname{Tp}(\mathrm{s})$ & 5 & 7 & 9 & 11 & 13 & 15 \\
\hline Hs (m) & 7 & 9 & 11 & 13 & 15 & 17 \\
\hline $4,5 \quad 5,5$ & & & & 27,15 & 17,19 & 10,08 \\
\hline $3,5 \quad 4,5$ & & & 21,18 & 22,33 & 13,09 & 7,35 \\
\hline $2,5 \quad 3,5$ & & 12,65 & 15,08 & 17,51 & 8,99 & 4,62 \\
\hline $1,5 \quad 2,5$ & 5,16 & 7,07 & 8,98 & 12,69 & 4,89 & 1,88 \\
\hline $0,5 \quad 1,5$ & 2,01 & 2,47 & 2,93 & 4,55 & 6,17 & \\
\hline
\end{tabular}

\section{Energies annuelles produites (MWh)}

\begin{tabular}{|c|c|c|c|c|c|c|}
\hline \multirow{2}{*}{ Tp (m) } & 5 & 7 & 9 & 11 & 13 & 15 \\
\hline & 7 & 9 & 11 & 13 & 15 & 17 \\
\hline $4,5 \quad 5,5$ & & & & 1,04 & 1,43 & 0,99 \\
\hline $3,5 \quad 4,5$ & & & 0,85 & 2,67 & 2,86 & 1,09 \\
\hline $2,5 \quad 3,5$ & & 0,94 & 1,97 & 4,94 & 3,48 & 0,83 \\
\hline $1,5 \quad 2,5$ & 0,27 & 1,72 & 3,54 & 8,34 & 2,80 & 0,39 \\
\hline $0,5 \quad 1,5$ & 0,28 & 1,67 & 3,70 & 5,04 & 1,97 & \\
\hline
\end{tabular}

Tableau 5. Résultats de production d'énergie du système à flotteur pilonnant

\begin{tabular}{lllll}
\hline Niveau de marée & $\begin{array}{l}\text { Coefficient de } \\
\text { pondération }\end{array}$ & $\begin{array}{l}\text { Puissance annuelle } \\
(\mathrm{kW})\end{array}$ & $\begin{array}{l}\text { Énergie annuelle } \\
(\mathrm{MWh})\end{array}$ & $\begin{array}{l}\text { Rendement } \\
(\%)\end{array}$ \\
\hline Niveau de basse mer $(1,45 \mathrm{~m})$ & 0,3 & 4,51 & 39,48 & 63,1 \\
Niveau de mi-marée $(3,07 \mathrm{~m})$ & 0,4 & 6,51 & 57,03 & 57,1 \\
Niveau de pleine mer $(4,75 \mathrm{~m})$ & 0,3 & 7,27 & 63,69 & 46,4 \\
\hline Sommation des trois niveaux & & 6,14 & 53,76 & 53,8 \\
\hline
\end{tabular}

\subsection{Coût de l'énergie}

Le coût de l'énergie est calculé en rapportant l'ensemble des coûts sur la durée de 20 ans à la production annuelle totale sur cette même durée, avec un taux d'actualisation retenu de $10 \%$. Pour un système à flotteur pilonnant de $5 \mathrm{~m}$ de long, d'un coût initial de $1 \mathrm{M} €$ incluant le CAPEX et le coût de démantèlement, et avec un OPEX fixé à un taux $10 \%$ du CAPEX, le coût de l'énergie produite la moins chère revient à $360 € / M W h$ pour la configuration optimale d'une demi-largeur de $2,5 \mathrm{~m}$, avec un tirant d'eau de 0,75 $\mathrm{m}$ et un dégagement de $3 \mathrm{~m}$ par rapport à la digue. La mise en place d'un système sur une durée de 30 ans, avec un taux d'actualisation réduit à $8 \%$, conduit à un coût d'énergie la moins chère de 275 €/MWh, diminuant ainsi le coût de l'énergie de 23,6 \%. 


\section{XVèmes Journées Nationales Génie Côtier - Génie Civil \\ La Rochelle, 29 au 31 mai 2018}

\section{Résultats du système oscillant à batteur inversé}

\subsection{Quantité d'énergie produite}

Le calcul de la puissance produite par le système est réalisé au point $n^{\circ} 4$, positionné devant l'extrémité de la digue à une profondeur de $5 \mathrm{~m} \mathrm{CM}$, par l'exploitation de la matrice de puissance fournie par l'ECN et des couples de houle ( $H s, T p$ ) obtenus en tranche 2 et utilisés comme paramètres d'entrée pour les trois niveaux de marée.

Tableaux 6, 7, 8 et 9. Puissances produites (en kW) et énergies annuelles (en MWh)

6. Puissances produites $(\mathrm{kW})$ à basse mer

\begin{tabular}{|cr|cccccc|}
\hline \multicolumn{2}{|c|}{ Tp (s) } & 5 & 7 & 9 & 11 & 13 & 15 \\
Hs (m) & 7 & 9 & 11 & 13 & 15 & 17 \\
\hline 4,5 & 5,5 & & & & 1761 & 1559 & 1305 \\
3,5 & 4,5 & & & 843 & 1257 & 1129 & 558 \\
2,5 & 3,5 & & 255 & 480 & 878 & 761 & 678 \\
1,5 & 2,5 & 127 & 184 & 214 & 415 & 467 & 428 \\
$\mathbf{0 , 5}$ & $\mathbf{1 , 5}$ & 75 & 51 & 95 & 104 & 170 & \\
\hline
\end{tabular}

\section{Puissances produites $(\mathrm{kW})$ à pleine mer}

\begin{tabular}{|cr|cccccc|}
\hline \multicolumn{2}{|c|}{ Tp (s) } & 5 & 7 & 9 & 11 & 13 & 15 \\
\multicolumn{2}{|c|}{ Hs (m) } & 7 & 9 & 11 & 13 & 15 & 17 \\
\hline 4,5 & 5,5 & & & & 526 & 383 & 222 \\
3,5 & $\mathbf{4 , 5}$ & & & 596 & 526 & 467 & 394 \\
2,5 & 3,5 & & 255 & 375 & 526 & 486 & 482 \\
$\mathbf{1 , 5}$ & $\mathbf{2 , 5}$ & 127 & 184 & 293 & 415 & 383 & 365 \\
$\mathbf{0 , 5}$ & $\mathbf{1 , 5}$ & 30 & 73 & 97 & 169 & 222 & \\
\hline
\end{tabular}

7. Puissances produites $(\mathrm{kW})$ à mi-marée

\begin{tabular}{|c|c|c|c|c|c|c|}
\hline \multirow{2}{*}{ Hs (m) } & 5 & 7 & 9 & 11 & 13 & 15 \\
\hline & 7 & 9 & 11 & 13 & 15 & 17 \\
\hline $4,5 \quad 5,5$ & & & & 1135 & 910 & 698 \\
\hline $3,5 \quad 4,5$ & & & 843 & 1007 & 910 & 698 \\
\hline $2,5 \quad 3,5$ & & 326 & 585 & 878 & 761 & 678 \\
\hline $1,5 \quad 2,5$ & 127 & 184 & 293 & 526 & 467 & 449 \\
\hline $0,5 \quad 1,5$ & 75 & 51 & 95 & 169 & 222 & \\
\hline
\end{tabular}

\section{Energies annuelles produites (MWh)}

\begin{tabular}{|c|c|c|c|c|c|c|c|}
\hline \multicolumn{2}{|c|}{$T p(s)$} & 5 & 7 & 9 & 11 & 13 & 15 \\
\hline \multicolumn{2}{|c|}{ Hs (m) } & 7 & 9 & 11 & 13 & 15 & 17 \\
\hline 4,5 & 5,5 & & & & 46,74 & 73,83 & 54,04 \\
\hline 3,5 & 4,5 & & & 33,06 & 121,90 & 171,17 & 60,60 \\
\hline 2,5 & 3,5 & & 21,28 & 68,52 & 244,70 & 238,76 & 76,66 \\
\hline 1,5 & 2,5 & 12,17 & 56,43 & 113,91 & 359,57 & 218,21 & 48,81 \\
\hline 0,5 & 1,5 & 14,96 & 49,66 & 128,93 & 183,65 & 74,30 & \\
\hline
\end{tabular}

Les résultats d'énergies annuelles montrent que le système à batteur inversé produit le plus d'énergie (359,57 MWh/an) pour le couple de houle (2 m, $12 \mathrm{~s})$. En sommant l'ensemble des énergies produites pour les couples de houle ( $H s, T p$ ), on obtient une énergie annuelle totale de 2521 MWh pour un flap oscillant d'une largeur de $20 \mathrm{~m}$.

Cependant, pour des conditions de faibles niveaux d'eau, plus de la moitié de la hauteur du flap oscillant, serait toujours émergée, ce qui doit poser des problèmes et rendre sans doute le système inopérant. C'est pourquoi, l'hypothèse d'un arrêt de fonctionnement du flap pour les faibles niveaux de marée est retenue. Le système ne semble pouvoir fonctionner qu'au-dessus d'un niveau médian (2,25 m) entre les niveaux de mi-marée (3,07 $\mathrm{m})$ et de basse mer $(1,45 \mathrm{~m})$; ce qui se traduit par la prise en compte uniquement des niveaux de mi-marée et de pleine mer dans la suite de l'analyse. Dans ces conditions d'exploitation, l'énergie annuelle totale est de 1701 MWh pour un rendement du système qui avoisine les $62 \%$ au point de sortie $n^{\circ} 4$ du site (voir tableau 10 ). 


\section{Thème 5 - Énergies et ressources marines}

Tableau 10. Résultats de la production d'énergie du système oscillant à batteur inversé en ne retenant que les niveaux de mi-marée et de pleine mer (point $n^{\circ} 4$ du site)

\begin{tabular}{lllll}
\hline Niveau de marée & $\begin{array}{l}\text { Coefficient de } \\
\text { pondération }\end{array}$ & $\begin{array}{l}\text { Puissance annuelle } \\
(\mathrm{kW})\end{array}$ & $\begin{array}{l}\text { Énergie annuelle } \\
(\mathrm{MWh})\end{array}$ & $\begin{array}{l}\text { Rendement } \\
(\%)\end{array}$ \\
\hline Niveau de basse mer $(1,45 \mathrm{~m})$ & 0,3 & $X$ & $X$ & $X$ \\
Niveau de mi-marée $(3,07 \mathrm{~m})$ & 0,4 & 312,67 & 2739,01 & 98,1 \\
Niveau de pleine mer $(4,75 \mathrm{~m})$ & 0,3 & 2016,86 & 230,24 & 77,4 \\
\hline Sommation des trois niveaux & & 194,13 & 1700,66 & 61,9 \\
\hline
\end{tabular}

\subsection{Coût de l'énergie}

Les coûts de l'énergie sont présentés pour les durées d'exploitation de 20 et 30 ans dans le tableau 11. L'ensemble de ces coûts sont calculés pour la mise en place d'une seule unité de production. En créant un parc de plusieurs unités, il pourrait être possible de mutualiser les coûts d’installation et de maintenance, réduisant ainsi le coût de l'énergie.

Tableau 11. Résultats de coût de l'énergie pour les durées d'exploitation du système oscillant à batteur inversé en ne retenant que les niveaux de mi-marée et de pleine mer

\begin{tabular}{llc}
\hline Coût initial (CAPEX et démantèlement) & & $5 \mathrm{M}$ \\
Coût de réparation et de maintenance (OPEX) & & $10 \%$ \\
Durée d'exploitation & $20 \mathrm{ans}$ & $30 \mathrm{ans}$ \\
Taux d'actualisation & $10 \%$ & $8 \%$ \\
Soit un coût total de & $9,68 \mathrm{M} €$ & $11,08 \mathrm{M} €$ \\
\hline Énergie totale sur la durée d'exploitation & $34 \mathrm{GWh}$ & $51 \mathrm{GWh}$ \\
\hline Coût de l'énergie (COE) & $285 € / \mathrm{MWh}$ & $217 € / \mathrm{MWh}$ \\
\hline
\end{tabular}

\section{Discussion et perspectives}

\subsection{Limites et comparaison des systèmes}

Le système à flotteur pilonnant proposé en matériaux composites de forme carrée, d’une longueur de $5 \mathrm{~m}$ et d'une demi-largeur de 2,5 m, justifie de retenir dans l'analyse un tirant d'eau de $0,75 \mathrm{~m}$. Des calculs supplémentaires pourraient être envisagés pour optimiser ce paramètre avec des matériaux de masses et formes différentes. Les résultats obtenus par le système à batteur inversé peuvent être considérés comme surestimés, en raison de la non-prise en compte des phénomènes visqueux et du sur-dimensionnement du flap vis-à-vis de la profondeur d'eau au point $\mathrm{n}^{\circ} 4$. L'émergence importante du flap oscillant d'une demi-hauteur pour les faibles niveaux d'eau doit réduire la production d'énergie et peut-être de la stopper. Les résultats de coûts de l'énergie des systèmes houlomoteurs étudiés sont présentés dans le tableau 12. L'écart relatif de coûts est de l'ordre de $20 \%$, avec des coûts de l'énergie plus favorable pour le système à batteur inversé, variant de $285 €$ /MWh sur une durée d'exploitation de 20 ans à 217 €/MWh sur 


\section{XVèmes Journées Nationales Génie Côtier - Génie Civil La Rochelle, 29 au 31 mai 2018}

une durée de 30 ans. Avec ces durées d'exploitation, les coûts de production de l'énergie du système sont proches de ceux des dispositifs éoliens offshore (ADEME, 2016).

Tableau 12. Comparaison des coûts de l'énergie des systèmes houlomoteurs oscillants à flotteur pilonnant et à batteur inversé en fonction de la durée d'exploitation

\begin{tabular}{lll}
\hline Coût de l'énergie des systèmes (COE) & 20 ans d'exploitation & 30 ans d'exploitation \\
\hline Coût de l'énergie du système à flotteur pilonnant & $360 € / M W h$ & $275 € / M W h$ \\
\hline Coût de l'énergie du système à batteur inversé & $285 € / M W h$ & $217 € / M W h$ \\
\hline Écart relatif : flotteur pilonnant vs batteur inversé & $20,8 \%$ & $21,1 \%$ \\
\hline
\end{tabular}

\subsection{Incertitudes et perspectives}

a) Les coûts initiaux des systèmes estimés à $1 \mathrm{M} €$ pour le flotteur et à 5 M€ pour le batteur restent incertains ; ils devront être ajustés pour des systèmes aboutis. Seule l'expérience accumulée dans le développement et la maintenance des technologies permettra une approximation réaliste du coût de l'énergie (SHARP et al., 2015). La mise en place de prototypes et de démonstrateurs doit permettre d'évaluer plus précisément les différents coûts des systèmes houlomoteurs dans les années à venir, avec des coûts qui sont amenés à baisser de près de 40 \% à l'horizon 2030 et devrait diminuer encore de moitié entre 2030 et 2050 (IRENA, 2015). Une approche basée sur des économies d'échelle afin de réduire les coûts réels, est envisageable en installant des parcs de plusieurs unités de production, afin de mutualiser les installations, comme les raccordements offshore et onshore.

b) D'autre part, les résultats de coûts de production d'énergie obtenus dans l'analyse s’appuient sur des modélisations numériques de systèmes oscillants, qui ont été développées à partir d'hypothèses simplifiées et d'essais en modèles réduits. Les modèles numériques induisent en effet des incertitudes vis-à-vis des comportements réels des systèmes en grandeur nature implantés sur site. De même, la modélisation des interactions houle-structure des systèmes houlomoteurs repose sur des théories linéarisées, comme la théorie potentielle. En réalité, il existe des effets non-linéaires atténuateurs, qui ont pour origines les non-linéarités mécaniques et de la surface libre (formes des spectres, déferlements turbulents), et les effets visqueux entre les composants des systèmes. Ces actions perturbatrices ont pour effet de contraindre les mouvements des systèmes et de contribuer à réduire les rendements.

c) Une autre part d'incertitude est présente dans l'analyse ; il s’agit des incertitudes liées à la variabilité de la ressource qu'il est souvent délicat d'estimer sur le long terme à partir des bases de données numériques de houle. Une étude complémentaire sur le sujet est proposée pour qualifier les variabilités intra-annuelles et inter-annuelles essentielles pour définir le potentiel prédictible, et évaluer le type de système houlomoteur le plus approprié au site d'Esquibien (CARBALLO et al., 2015). 


\section{Thème 5 - Énergies et ressources marines}

\section{Références bibliographiques}

ADEME (2016). Coût des énergies renouvelables en France, Collection Faits \& Chiffres, $52 \mathrm{p}$.

BAUDRY V., MARRONE S., BABARIT A., LE TOUZÉ D., CLÉMENT A.H. (2015). Power matrix assessment and extreme loads estimation on a flap type wave energy converter in front of a dike, Proceeding of the 11th European Wave and Tidal Energy Conference, Nantes, France, 6-11 September 2015.

CARBALLO R., SANCHEZ M., RAMOS V., FRAGUELA J.A., IGLESIAS G. (2015). The intra-annual variability in the performance of wave energy converters: A comparative study in $N$ Galicia (Spain), Energy, Vol. 82, pp 138-146. https://doi.org/10.1016/i.energy.2015.01.020

DALTON G., ALCORN R., \& LEWIS T. (2010). Operational expenditure costs for wave energy projects, insurance and site rent, ICOE 2010, $3^{\text {rd }}$ International Conference on Ocean Energy, 6 October, Bilbao.

EQUIMAR (2011). Deliverable D7.2.1, Procedures for Economic Evaluation, Equitable Testing and Evaluation of Marine Energy Extraction Devices in terms of Performance, Cost and Environmental Impact, 15 p.

GUANCHE R., DE ANDRÉS A.D., SIMAL P.D., VIDAL C., LOSADA I.J. (2014). Uncertainty analysis of wave energy farms financial indicators, Renewable Energy, Vol. 68, pp. 570-580. https://doi.org/10.1016/j.renene.2014.02.046

IRENA (2015). Renewable power generation costs in 2014, 164 p.

MICHARD B., COSQUER E., DUFOUR G. (2014). Projet EMACOP : évaluation du potentiel houlomoteur de 22 sites français, XIII ${ }^{\text {èmes }}$ JNGCGC, Dunkerque, pp 751-758. https://doi.org/10.5150/jngcgc.2014.082

MICHARD B., COSQUER E., MALLÉGOL A., COIGNARD J., FILIPOT J.F., KPOGO-NUWOKLO K.A., OLAGNON M., SERGENT P. (2016). Projet EMACOP : Caractérisation des vagues et du potentiel houlomoteur des sites d'Esquibien et de Saint-Guénolé par simulation numérique, XIV èmes JNGCGC, Toulon, pp 497-508. https://doi.org/10.5150/jngcgc.2016.056

NEUVÉGLISE S., MARIN F., PERRET G., SMAOUI H., SERGENT P. (2017). Numerical and Physical Modeling of a Quayside Wave Energy Recovery System, Proceedings of 12th European Wave and Tidal Energy Conference, Cork, Ireland, 27 August-1 September 2017.

RUSU L., ONEA F. (2015). Assessment of the performances of various wave energy converters along the European continental coasts, Energy, Vol. 82, pp 889-904. https://doi.org/10.1016/j.energy.2015.01.099

SHARP C., DUPONT B. (2015). Analysis of WEC Array Economics: Current State-ofthe-Art and Future Needs, Proceeding of the 11th European Wave and Tidal Energy Conference, Nantes, France, 6-11 September 2015. 